\section{Pulmonary barotrauma in submarine escape training}

\author{
Caroline R Broome, Lionel J Jarvis, Robin \\ J Clark
}

\begin{abstract}
A 25 year old submariner sustained pulmonary barotrauma during simulated submarine escape. The unusual features of the distribution of pleural and extrapleural air are described and explained.
\end{abstract}

(Thorax 1994;49:186-187)

As part of training for service in submarines, personnel are regularly required to rehearse simulated escape from a pressurised chamber well below the surface. We describe the unusual radiographic appearances of a submariner who developed extensive air tracking following pulmonary barotrauma during such an ascent.

\section{Case report}

A previously healthy 25 year old man underwent submarine escape tank training following stringent screening which included physical examination, spirometric measurements, and normal inspiratory and expiratory chest radiographs. The subject passed through a pressurised airlock 18 metres below the surface of a water tower. He made a buoyant ascent through the water and surfaced with a loud gasp, apparently unconscious. $\mathrm{He}$ was removed rapidly from the water and suffered a convulsion. A clinical diagnosis was made of cerebral arterial gas embolism secondary to pulmonary barotrauma and he was placed in a therapeutic dry recompression chamber with a medical attendant. He was compressed rapidly to 50 metres of water (six bar) and regained consciousness within four minutes of his ascent. $\mathrm{He}$ was decompressed to atmospheric pressure over four hours, receiving intermittent hyperbaric oxygen therapy. On removal from the chamber he was neurologically normal, but there were clinical features of a right pneumothorax and subcutaneous emphysema. He was not distressed, so further management was delayed until transfer to hospital.

The initial chest radiograph (fig 1) showed bilateral pneumothoraces, pneumomediastinum, subcutaneous emphysema, and bilateral pockets of air loculated between the parietal pleura and the diaphragmatic surfaces. Subsequent plain abdominal radiographs showed additional free gas in the retroperitoneal and intraperitoneal spaces. The larger right pneumothorax was relieved by intercostal tube drainage and simple aspiration was undertaken on the left. A radiograph taken in the head down position confirmed that the subpleural air pockets remained fixed above the diaphragm, with no evidence of movement into the peritoneal cavity (fig 2). A subsequent computed tomographic scan confirmed the presence of subpleural air collections with dependent fluid within. Progressive clinical improvement was impeded by a right basal pneumonia which responded to standard antibiotic therapy. The chest radiographic appearances resolved, with subpleural collections persisting for 20 days. A delayed radiograph taken five months after the incident showed residual pleural scarring at the right base. $\mathrm{He}$ has returned to work but is no longer considered fit for submarine escape tank training.

\section{Discussion}

Before submarine escape tank training, trainees are taught to exhale continuously during

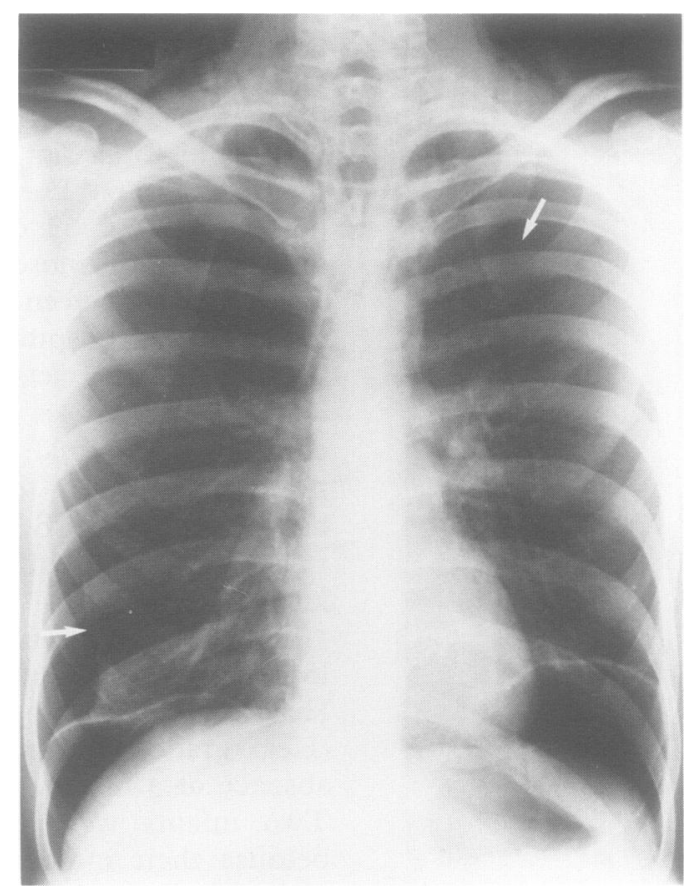

Figure 1 Chest radiograph on admission showing bilateral pneumothoraces (arrows), pneumomediastinum, subcutaneous emphysema, and extrapleural air.

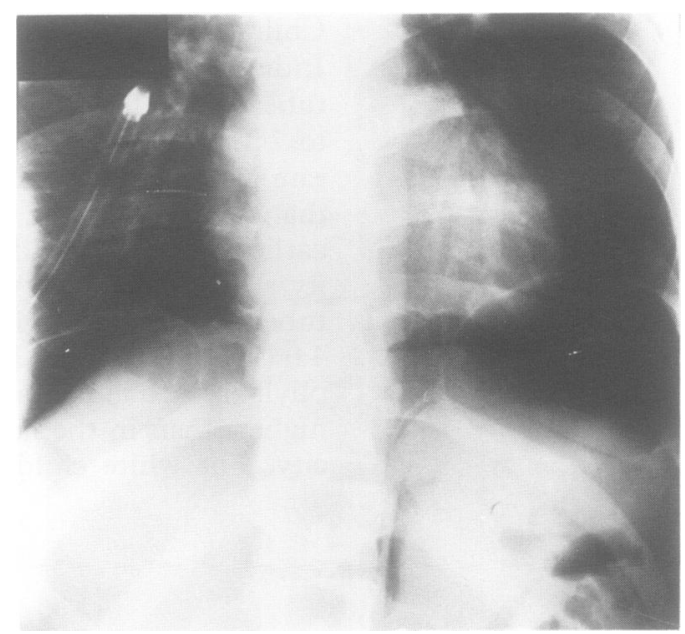

Figure 2 Radiograph taken with patient in head down position showing retention of air above the diaphragm. 
ascent to vent the intrathoracic gas which expands during rapid decompression. This subject appears to have maintained partial glottic or pharyngeal closure, resulting in the development during ascent of a markedly positive instantaneous intrathoracic pressure relative to ambient pressure. This caused pulmonary barotrauma, with leakage of air from one or more airspaces into the pulmonary parenchyma. ${ }^{1}$ With continuing leakage under pressure the gas ruptured through multiple anatomical barriers, since none alone was able to accommodate the flux of gas demanded of such rapid expansion. It appears that gas tracked into the pulmonary vasculature, resulting in cerebral arterial gas embolism, into both pleural spaces, along the pulmonary pedicle to the mediastinum, then along the connective tissue continuum to the subcutaneous tissues of the neck. Gas also tracked down through the diaphragmatic hiatus to the retroperitoneal space, from which it ruptured into the peritoneal cavity. ${ }^{2}$ Most remarkably, gas passing through the hiatus appears to have dissected along the connective tissue plane attaching the parietal pleura to the dome of each hemidiaphragm. This tissue is usually sufficiently adherent at least to maintain adherence of the pleura to the hemidiaphragm during diaphragm contraction and is difficult to strip surgically. Having initiated a gas filled cavity, the expanding gas would have been aided by the Laplace effect (as when blowing up a child's balloon becomes progressively easier). The persistence of these gas filled cavities for over four weeks (despite the intermittent use of oxygen therapy) supports their location in a poorly perfused plane.

References to similar appearances can be found in the literature. In a review of submarine escape tank training incidents in the Royal $\mathrm{Navy}^{3}$ a 19 year old required recompression following loss of consciousness after a normal ascent. His chest radiograph after recompression showed identical bilateral basal air collec- tions to our case, though these were reported as cysts at the lung bases. The presence of the clearly defined opaque bands defining the extent of these cysts which extend to the chest wall and beyond the margin of the collapsed lung confirms that these cannot represent pulmonary air cavities, but must lie beneath the pleural surface. We suggest that this case represented an earlier presentation of this distribution of extrapleural air of similar aetiology.

Lillard and Allen ${ }^{4}$ reported the presence of radiolucent pockets of free air between the parietal pleura and diaphragm in neonates with respiratory distress; they proposed the "extrapleural air sign" to diagnose this appearance and to support the presence of an otherwise difficult assessment of a pneumomediastinum. Their description of the route taken by the air, and the justification for the site of the air collections, was also well emphasised. This has subsequently been described in reviews of mediastinal emphysema in the newborn ${ }^{56}$ but we can find no reference to this appearance in the adult.

The presence of this pattern of extrapleural air distribution is an extremely rare manifestation of pulmonary barotrauma. The circumstances in our case, however, were well beyond normal clinical practice. Extreme changes in pressure with rapid gas expansion are clearly necessary to strip the parietal pleura from the diaphragmatic surface, and to provide an additional anatomical plane within which gas can collect.

1 Strauss RH. Diving medicine. Am Rev Respir Dis 1979;119:1001-23.

2 Machlin CC. Transport of air along sheaths of pulmonic blood vessels from alveoli to mediastinum. Arch Intern Med 1939;64:913-26.

3 Elliott DH, Harrison JAB, Bernard EEP. Clinical and radiological features of 88 cases of decompression barotrauma Underwater Physiol 1978;6:527-36.

4 Lillard RL, Allen RP. The extrapleural air sign in pneumomediastinum. Radiology 1965;85:1093-8.

5 O'Gorman LD, Cottingham RA, Sargent EN, O'Loughlin 'Gorman LD, Cottingham RA, Sargent EN, O'Loughlin
BJ. Mediastinal emphysema in the newborn: a review and BJ. Mediastinal emphysema in the newborn: a review and
description of the new extrapleural gas sign. Dis Chest 1968;53:301-8.

6 Felson B. The mediastinum. Semin Roentgenol 1969;4:41-58. 